D. Mrchlin. Uber die Wirkung der Elektrolyten auf die Synthese der Lactose (Jahr 1940).

I. I. Nitzescu et G. Nicolat. C. R. Soc. Biol., 1924, XCI, 1462.

T. R. Parson. Biological Chemistry, IV. Edit. Cambridge.

Ch. Porcher. L'origine du lactose. Arch. Int. de Phys., vol. VIII, 1909.

Ch. Porcher. In a Traité de physiologie norm. et pathol. ". Vol. XI, Paris, 1927, p. 293. Masson, édit.

M. Ragno. Atti Acc. Peloritana, Messina, 39, 1937.

Rondonr. Biochimica, Torino, U. T. E. T.

R. Rossi, Boll. Soc. It. Biol. Sper., 8, 1933.

E. Savini. Il Latte, Milano, Hoepli, édit., 1942, 41.

P. SHARP. Jahr. Band CVII, 1938.

M. Spizzi. La Clinica Vet., Milano, 1936.

\title{
L'ANALYSE BACTÉRIOLOGIQUE DES LAITS CONCENTRÉS SUCRÉS. \\ PRÉSENCE DE STAPHYLOCOQUES PATHOGÈNES DANS CERTAINS D'ENTRE EUX
}

\author{
par
}

\author{
René BUTtiAUX et Lucie LESNE
}

Il est de connaissance assez récente que certains staphylocoques déterminent par absorption digestive, chez l'homme, des toxi-infections alimentaires. DolmaN et Mildward BAyLISS [1] ont montré que ces germes possèdent des toxines thermostables qui sont responsables des vomissements et de la diarrhée. Ils ont réussi à les isoler et à reproduire expérimentalement, chez le chat, les troubles constatés chez l'homme.

La toxi-infection suit de quelques heures, 8, 10, 12 ou 24 heures l'absorption de l'aliment contaminé. Les aliments responsables sont, dans la majorité des cas, les laits de conserve ou crus, les crèmes de laits, les crèmes glacées, les viandes conservées. Les staphylocoques pathogènes paraissent être amenés dans ces produits, le plus souvent, par des contaminations secondaires, au cours de la préparation ou de la fabrication. Parfois ils se rencontrent dans les laits de vaches atteintes de mammites. Les cas publiés, sont, maintenant, assez nombreux, et nous signalerons en particulier les communications de BАмM [2] (laits, crèmes, crèmes glacées) ; de Slater [3] (corned-beef) ; de Worral [4] qui a isolé d'assez nombreux staphylocoques pathogènes dans les diarrhées ou les vomissements des enfants; de SEwit [5] qui considère que 13,5\% des cas de diarrhées observés à Dublin en 1942-43 étaient dus au staphylocoque ; de Lawson [6].

Durant l'été 1945, nous avons eu l'occasion d'observer des faits 
semblables. Certains laits concentrés sucrés d'origine étrangère, avaient été distribués pour l'alimentation des nourrissons. Leur absorption a déterminé des troubles graves et malheureusement parfois mortels chez une importante proportion d'entre eux. L'Institut Pasteur de Lille a été chargé de l'étude bactériologique des laits incriminés et nous y avons isolé, dans de nombreux cas, des staphylocoques pathogènes, qui étaient responsables des toxiinfections observées.

Il nous paraît donc intéressant de résumer ici quelques points importants de technique qui permettent facilement de dépister et de diagnostiquer cette souillure alimentaire, dans les laits concentrés sucrés.

Le problème se divise en deux parties :

10 Isoler les staphylocoques;

$2^{\circ}$ Préciser s'il s'agit d'un staphylocoque pathogène ou d'un staphylocoque saprophyte.

Ces recherches ne constituent qu'une partie de l'analyse bactériologique d'un lait concentré sucré. D'autres germes sont en effet susceptibles d'altérer sa qualité.

C'est pourquoi il nous paraît indispensable de préciser, ici, les différents points importants de la technique d'une analyse bactériologique complète de lait concentré sucré.

\section{A. Analyse bactériologique du lait concentré sucré}

Nous pratiquons les opérations suivantes :

La boîte est placée à l'étuve à $37^{\circ}$ durant 48 heures. Nous recherchons à ce moment les signes de «bombage ».

Nous l'ouvrons aseptiquement après nettoyage des parois à l'alcool à $95^{\circ}$ et flambage rapide. Le lait est alors dilué dans 2 fois son volume d'eau stérile.

Sur le lait dilué, nous effectuons la série des recherches suivantes :

$1^{\circ}$ Numération des germes en ensemençant $1 \mathrm{~cm}^{3}, 1 / 10^{\mathrm{e}} \mathrm{cm}^{3}$, $1 / 100^{\mathrm{e}} \mathrm{cm}^{3}, 1 / 1.000^{\mathrm{e}} \mathrm{cm}^{3}$ sur lait digéré gélosé en boîte de Pétri (milieu de Guittonneau). Une série de boîtes est mise, à incuber à $37^{\circ}$ durant 48 heures, une autre série à $30^{\circ}$ durant 72 heures. Les lectures faites au bout de ces temps respectifs indiquent le nombre total de germes vivants.

$2^{\circ}$ Recherche et numération du B. Coli. Nous employons en ce moment, en même temps, pour un même lait, 3 techniques qui ne diffèrent que par la nature des milieux de culture :

Le milieu bilié au vert-brillant.

Le milieu peptoné à la trypaflavine [7].

Le bouillon lactosé dit “Lactose-Broth " américain. 
Les deux premiers et surtout le second bloquent assez bien le développement des autres germes que le $B$. Coli. Le troisième, qui n'a pas d'effet inhibiteur sur la flore habituelle du lait, nous est très utile pour la recherche et la numération des staphylocoques. Nous y reviendrons tout à l'heure.

Nous ensemençons successivement sur ces divers milieux :

5 tubes de chaque variété avec $10 \mathrm{~cm}^{3}$ de lait dilué.

1 tube de chaque variété avec $1 \mathrm{~cm}^{3}$ de lait dilué.

1 tube de chaque variété avec $1 / 10^{\mathrm{e}} \mathrm{cm}^{3}$ de lait dilué.

1 tube de chaque variété avec $1 / 100^{\mathrm{e}} \mathrm{cm}^{3}$ de lait dilué.

1 tube de chaque variété avec $1 / 1.000 \mathrm{~cm}^{3}$ de lait dilué.

Les tubes sont portés à l'étuve à $37^{\circ}$. Les cultures et fermentations sont observées après 48 heures. Chaque tube avec ou sans gaz, mais où une culture manifeste s'est produite est repiqué sur un tube d'eau peptonée riche en tryptophane (Tryptone Difeo). L'indol y est recherché après 72 heures d'incubation à 37 o.

$3^{\circ}$ Recherche et numération des germes putrides. Le lait, à cet effet, est ensemencé, sur des ballons ou tubes contenant du bouillonpeptoné nutritif ordinaire, de la façon suivante :

$10 \mathrm{~cm}^{3}$ de lait dans un ballon de $50 \mathrm{~cm}^{3}$ de bouillon;

$1 \mathrm{~cm}^{3}$ de lait, $1 / 10^{\mathrm{e}} \mathrm{cm}^{3}, 1 / 100^{\mathrm{e}} \mathrm{cm}^{3}, 1 / 1.000^{\mathrm{e}} \mathrm{cm}^{3}$ dans des tubes de $10 \mathrm{~cm}^{3}$ de bouillon.

A la partie supérieure de ces ballons et tubes, est placée une languette de papier filtre imbibée d'une solution d'acétate de plomb et préalablement stérilisée à l'autoclave. Le petit fragment de papier est glissé entre le bouchon de coton et le verre, et maintenu ainsi à $1 \mathrm{~cm}$. environ au-dessus du niveau du liquide.

Les liquides ensemencés sont portés à l'étuve à $37^{\circ}$ durant 48 heures. La présence d' $\mathrm{H}^{2} \mathrm{~S}$ est indiquée par le noircissement $\mathrm{du}$ papier à l'acétate de plomb.

$4^{\circ}$ Recherche et numération des germes anaérobies. Nous utilisons en même temps deux techniques.

La première, dérivée de l'analyse bactériologique de l'eau, met en évidence le $B$. perfringens. Nous chauffons $20 \mathrm{~cm}^{3}$ de lait dilué au thermostat à $75-80^{\circ}$ durant 5 minutes. Nous ensemençons alors $10 \mathrm{~cm}^{3}$, puis $5 \mathrm{~cm}^{3}$ de ce lait dans de grands tubes contenant $20 \mathrm{~cm}^{3}$ de gélose glucosée sulfitée à l'alun de fer, fondus au bain-marie et ramenés à une température de $65^{\circ}$ environ. Dès que le mélange du lait et de la gélose est obtenu par agitation brutale (éviter les bulles), on refroidit rapidement sous un jet d'eau froide. Les tubes sont placés à l'étuve à $37^{\circ}$. Au bout de 24 à 48 heures, le $B$. perfringens donne de belles colonies noires. 
La seconde est la technique de WeinzirL [8] ; elle est simple et efficace. On répartit dans 5 tubes stériles, $5 \mathrm{~cm}^{3}$ de lait par tube. On chauffe 10 minutes à $80^{\circ}$. On coule, à la surface de chacun d'entre eux, un peu de paraffine liquéfiée par chauffage. On laisse refroidir et on porte à l'étuve à $37^{\circ}$ pendant 3 ou 4 jours. La présence de gaz se manifeste par de grosses bulles qui apparaissent sous le disque de paraffine et le font remonter, s'ils sont très abondants. Un développement gazeux net dans 2 des 5 tubes permet, a priori, de considérer le lait étudié comme de mauvaise qualité bactériologique. Une coloration de Gram faite à ce moment donne des précisions sur la flore qui s'est développée.

$5^{\circ}$ Recherche des levures, torulas. Nous ensemençons $10 \mathrm{~cm}^{3}$, $2 \mathrm{~cm}^{3}, 1 \mathrm{~cm}^{3}$ de lait dilué sur 3 tubes d'eau de malt, que l'on porte à $30^{\circ}$ durant 3 jours. Des colorations sont faites à ce moment et des isolements pratiqués si nécessaire.

$6^{\circ}$ Recherche des germes du "lait visqueux" ou "filant". On répartit quelques $\mathrm{cm}^{3}$ de lait dilué dans un tube de lait écrémé stérilisé, amené à $p H$ 7,4 et contenant un peu d'une solution de rouge de phénol. On porte à l'étuve à $30^{\circ}$ durant 2 ou 3 ou 4 jours. On observe l'acidification ou l'alcalinisation par virage de l'indicateur. On se rend compte de la consistance du lait inoculé à ce moment.

$7^{\circ}$ Recherche des salmonella. Dans certains cas particuliers, où la clinique humaine fait suspecter une infection possible par des salmonella, il peut être nécessaire de rechercher ces germes dans les laits de conserve. Nous employons les techniques suivantes :

a) Enrichissement sur milieu de culture liquide. On a le choix entre le milieu de Muller-Kauffmann et le milieu américain au Sélénite dit "Selenit M ». On ensemence 10 ou $20 \mathrm{~cm}^{3}$ de lait sur ces milieux et l'on porte à l'étuve à $37^{\circ}$ durant 48 heures. Les salmonella se développent abondamment alors que les autres microbes sont bloqués dans leur culture;

b) Isolement sur milieux électifs à partir des milieux de culture enrichis. On aura recours soit au milieu gélosé de Wilson-Blair au citrate de bismuth ammoniacal, soit à la gélose " $\mathrm{S}$. S. " américaine. 1 ou 2 gouttes des milieux enrichis sont étalées à la surface de ces géloses. Les plaques sont portées à l'étuve à $37^{\circ}$. Au bout de 24 heures, les salmonella donnent sur S. S. des colonies incolores avec petit centre noir ; les $B$. Coli des colonies rouge framboise, toujours très rares. Après 24 à 48 heures, les salmonella se présentent sur milieu de Wilson-Blair sous la forme de colonies noires à halo et reflet métallique. 


\section{B. Recherche et isolement des staphylocoques}

I. Deux techniques peuvent étre employées. Nous signalerons celle qui nous fournit les meilleurs résultats et que nous employons maintenant de façon constante. Nous avons remarqué que le bouillon lactosé "lactose Broth" recommandé par la "Standard method" américaine pour l'analyse des eaux, était un très bon milieu de culture pour le staphylocoque. Nous prenons donc les tubes de lactose Broth qui nous ont servi pour la recherche du B. Coli. Nous faisons une coloration de Gram sur chacun d'entre eux qui a cultivé, et, par ailleurs, un isolement sur quelques tubes de gélose nutritive (glucosée à $1 \%$ ). Pour bien mettre en évidence la pigmentation des staphylocoques, nous utilisons avec gros avantage, dans la préparation de cette gélose nutritive, une peptone spéciale de la maison Difco, dénommée "tryptose ". Après 24 heures d'étuve à $37^{\circ}$ les colonies suspectes sont repiquées et identifiées. Nous avons de cette manière, naturellement, toutes les variétés de staphylocoques, aussi bien pathogènes que non pathogènes.

Chapman [9] a mis au point un milieu qui ne permet au premier passage que le développement des seuls staphylocoques pathogènes. C'est la gélose lactosée au bleu de bromo-thymol. Il suffit d'ensemencer directement le lait dilué à la surface d'une ou plusieurs boîtes de Pétri où a été coulé le milieu. On porte à l'étuve à $37^{\circ}$ durant 36 heures. A ce moment, les staphylocoques pathogènes donnent des colonies bien développées, nettes, muqueuses, généralement jaunâtres. L'écueil de la technique est la trop faible quantité de lait qui peut être ensemencée. Dans les cas où le lait étudié contient peu de staphylocoques, la recherche risque donc d'être négative (1).

II. Recherche du pouvoir pathogène du staphylocoque isolé. Il existe 2 variétés importantes de staphylocoques :

Ceux qui possèdent un pouvoir pathogène.

Ceux qui ne sont que des saprophytes.

On admettait jusqu'ici que la pigmentation des cultures était suffisante pour différencier ces deux variétés. On sait aujourd'hui qu'il n'en est rien. S'il est vrai que presque tous les staphylocoques dorés possèdent une virulence certaine, il existe des staphylocoques blanes qui sont doués de la même propriété. Le moyen de diagnostic indiscutable est l'inoculation à l'animal. En pratique, ces recherches sont souvent irréalisablẹs. On a donc essayé de mettre au point quelques tests biochimiques qui peuvent y suppléer.

(1) Depuis la rédaction de cet article nous utilisons un nouveau procédé d'isolement mis au point par Chapman. Cet auteur utilise la propriété des staphylocoques de se développer à des concentratious chlorurées sodiques de $75 \%$. Nous déerirons cette technique à la Soc. Fr. de Microb. (séance d'avril 1947). 
Un staphylocoque pathogène se reconnaît aux caractères suivants :

$1^{\circ} \mathrm{Il}$ est généralement pigmenté en jaune doré, mais certaines variétés blanches font exception;

$2^{\circ} \mathrm{Il}$ coagule plus ou moins rapidement mais toujours nettemen 7 le plasma sanguin;

$3^{\circ} \mathrm{Il}$ est hémolytique;

$4^{\circ}$ Il fermente énergiquement la mannite;

$5^{\circ}$ Cultivés au $2^{\mathrm{e}}$ passage sur gélose au violet de gentiane, après 36 heures d'étuve à $37^{\circ}$, les staphylocoques pathogènes donnent des colonies violettes ou pourpres, les staphylocoques non pathogènes des colonies blanches, ne concentrant pas le colorant à leur surface $[10,11]$. Chapman admet que dans $96 \%$ des cas, le test au violet est suffisant pour faire le diagnostic de la variété isolée.

STONE a montré, par ailleurs, que les staphylocoques responsables des toxi-infections intestinales, possèdent des propriétés particulières. Ensemencés sur un milieu gélosé-gélatiné, riche en extrait de viande, les staphylocoques fournissent en 24 heures à $37^{\circ}$ des colonies bien venues. On recouvre à ce moment la boîte de Pétri contenant le milieu cultivé avec une solution saturée de sulfate d'ammoniaque. En moins de 5 minutes, on voit les colonies de staphylocoques pathogènes s'entourer d'une zone claire auréolaire, d'autant plus grande que le germe est plus riche en toxines entérotropes. Il est utile de compléter cette recherche par l'étude des propriétés hémolytiques du germe isolé. On admet finalement qu'un staphylocoque nettement hémolytique, s'auréolant d'une zone claire sur milieu de Stone, doit être considéré comme responsable des toxi-infections intestinales.

Pour déterminer le pouvoir pathogène des staphylocoques, nous recherchons ces divers tests de la façon suivante :

$1^{\circ}$ Recherche de la pigmentation. La pigmentation est recherchée par culture sur gélose peptonée, légèrement glucosée (A.).

$2 \circ \mathrm{La}$ coagulase est étudiée par la méthode simplifiée de Lominski [13]. 1 trace de culture est ajoutée à 1 petit tube contenant $1 / 2 \mathrm{~cm}^{3}$ de plasma humain oxalaté, dilué dans 5 fois son volu $K$ lume de sérum physiologique à $8,5 \% \mathrm{o}$. On mélange et l'on met incuber à l'étuve à $37^{\circ}$. On recherche la coagulation après $1 / 2$ heure, puis 1 heure, 3 heures, 6 heures et 12 heures. La coagulase est d'autant plus active que la coagulation du plasma se produit plus rapidement.

$3^{\circ}$ L'hémolysine est recherchée par ensemencement sur une boîte de Pétri où l'on a coulé de la gélose nutritive additionnée de sang humain ou de lapin (mélange fait à $50^{\circ}$ juste avant l'emploi). 
La zone hémolysée qui entoure la culture après 24 heures d'étuve à $37^{\circ}$ est d'autant plus grande que l'hémolysine sécrétée par le staphylocoque est plus active.

$4^{\circ}$ La fermentation de la mannite est étudiée par ensemencement sur milieu mannité avec comme indicateur le rouge de phénol (B.). On met à l'étuve à $37^{\circ}$ durant 24 ou 36 heures. L'abaissement du $p \mathrm{H}$ est d'autant plus rapide et important que la fermentation de la mannite est plus grande.

$5^{\circ}$ Le test au cristal-violet est pratiqué par culture sur gélose lactosée au violet de gentiane (C.). On ensemence par points séparés une assez grosse quantité de staphylocoque isolé. On porte à l'étuve à $37^{\circ}$ durant 36 heures. Les staphylocoques pathogènes fournissent des colonies violettes ou pourpres.

Le manque actuel, en France, d'extrait de viande de bœuf, ne nous a pas permis d'étudier, d'une façon constante, la culture sur milieu de Stone (D.). Nous ne pouvons, par conséquent, tenir compte ici des résultats insuffisants que nous possédons à ce sujet.

III. Mise en évidence de staphylocoques pathogènes dans certains laits concentrés sucrés, d'origine étrangère. Au cours et à la suite des toxi-infections intestinales produites chez les nourrissons du Nord de la France par l'absorption de laits concentrés sucrés d'origine étrangère, nous avons eu à examiner 66 échantillons de ces produits, provenant de marques différentes ou de séries diverses dans une même marque. Dans 20 d'entre eux, nous avons isolé un staphylocoque, 14 fois, il s'agissait d'un staphylocoque nettement pathogène, responsable des accidents constatés chez les nourrissons. Pour isoler le staphylocoque, nous partons comme nous l'avons dit précédemment, des bouillons lactosés ensemencés avec les dilutions croissantes de lait concentré dilué.

Le tableau annexé montre nettement les indications utiles que nous ont fourni ces diverses recherches bactériologiques. Un point important mérite d'être signalé. Si dans la majorité des cas, les staphylocoques reconnus pathogènes étaient dorés, il en est trois qui fournissaient des colonies blanches, absolument exemptes de toute trace de pigmentation. On rencontre, par conséquent, dans les laits concentrés sucrés, des staphylocoques blancs qui sont doués de propriéts pathogènes certaines. Il est donc nécessaire de pratiquer, chaque fois, la recherche des tests biologiques que nous venons de signaler.

Par ailleurs, les numérations forcément approximatives que nous avons effectuées, montrent que la concentration en staphylocoques est variable d'un lait à l'autre. Si certains en contiennent 100.000 par litre, d'autres n'en renferment que 100. Pourtant, on 
R. BUtTiAUX ET LUCiE LESNe. - L'ANALYSE BACtériologiQue

\begin{tabular}{|c|c|c|c|c|c|c|c|}
\hline $\begin{array}{c}\text { Marques et estampes } \\
\text { des laits concentrés } \\
\text { sucrés }\end{array}$ & $\begin{array}{l}\text { Pig- } \\
\text { menta- } \\
\text { tion }\end{array}$ & $\begin{array}{c}\text { Coagu- } \\
\text { lase }\end{array}$ & $\begin{array}{c}\text { Hémo- } \\
\text { lyse }\end{array}$ & $\begin{array}{c}\text { Fer- } \\
\text { menta- } \\
\text { tion } \\
\text { de la } \\
\text { mam- } \\
\text { mite }\end{array}$ & \begin{tabular}{|c|} 
Culture \\
sur \\
gélose \\
au \\
cristal \\
violet
\end{tabular} & $\begin{array}{c}\text { Numéra- } \\
\text { tion } \\
\text { approxi- } \\
\text { mative } \\
\text { par } \\
1.000 \mathrm{~cm} 3 \\
\text { de lait } \\
\text { dilué }\end{array}$ & $\begin{array}{l}\text { Liqué- } \\
\text { faction } \\
\text { de la } \\
\text { gélatine }\end{array}$ \\
\hline B. $1.894 \quad \ldots \ldots \ldots$ & Doré & + & +++ & $t+t$ & + & 10.000 & + \\
\hline B. $105 \ldots \ldots$ & Doré & + & 0 & $+t$ & + & 10.000 & + \\
\hline B. $95 \ldots \ldots \ldots \ldots$ & Doré & + & 0 & $+t$ & + & 10.000 & + \\
\hline B. $165 \ldots \ldots \ldots \ldots$ & Doré & + & + & ++ & + & 10.000 & + \\
\hline B. $145 \ldots \ldots \ldots \ldots$ & Doré & + & 0 & + & + & 10.000 & + \\
\hline B. $175 \ldots \ldots \ldots$ & Doré & + & $+t$ & +++ & + & 100.000 & + \\
\hline M. A. $2 \ldots$ & Blane & 0 & 0 & \pm & 0 & 100 & 0 \\
\hline M. A. $1 \ldots \ldots \ldots$ & Blane & 0 & 0 & \pm & 0 & 100 & 0 \\
\hline M. A. 48 .. & Doré & & +++ & +++ & + & 100 & 0 \\
\hline M. A. $49 \ldots$ & Blane & 0 & 0 & \pm & 0 & 100 & 0 \\
\hline M. B. $96 \ldots \ldots \ldots$ & Doré & + & 0 & \pm & + & 10.000 & + \\
\hline A. S. $28 \ldots \ldots \cdots \cdots$ & Doré & + & $+t$ & $++t$ & + & 100 & + \\
\hline A. T. $13 \ldots \ldots$ & Doré & + & 0 & ++ & + & 10.000 & 0 \\
\hline P. 20 D.D $\ldots \ldots \ldots$ & Blane & 0 & 0 & \pm & 0 & 1.000 & 0 \\
\hline P. 23 D.D $\ldots \ldots \ldots$ & Blane & 0 & 0 & \pm & 0 & 1.000 & 0 \\
\hline R. 010 N.D. $j \ldots \ldots$ & Blane & + & $+t$ & $+t$ & $\div$ & 1.000 & + \\
\hline R. 014 N.D. . . . . & Blane & \pm & ++ & + & + & 100 & + \\
\hline R. T.I.N.D. . . W & Blane & \pm & ++ & + & + & 1.000 & + \\
\hline N. ARSMAW $\ldots$. & Blane & 0 & 0 & \pm & 0 & 1.000 & 0 \\
\hline P. H. $66552 \ldots \ldots \ldots$ & Doré & + & $++t$ & $++t$ & + & 10.000 & + \\
\hline & & & & & & & \\
\hline
\end{tabular}


trouve parfois, et c'est le cas du M. A.48, des staphylocoques très pathogènes, qui n'existent qu'en petit nombre. Il est impossible de prévoir si, même dans ce cas, le produit ingéré n'est pas aussi dangereux que celui riche en microbes. Il semble, en effet, que dans les intoxications alimentaires, la qualité du germe infectant est plus importante à envisager que sa quantité.

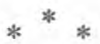

Une question importante reste à résoudre. D'où proviennent ces staphylocoques pathogènes ? Comment peuvent-ils se rencontrer dans les laits concentrés sucrés ? Nous ne voulons qu'esquisser, ici, ce problème étiologique. L'origine peut être double : la moins probable est celle qui ferait remonter la contamination à une souillure originelle du lait eru par un staphylocoque de mammite, par exemple, ou un staphylocoque du trayeur. La plus probable est celle qui admet la contamination du produit fini par des souillures des manipulateurs ou des appareils de fabrication. Il est important de signaler, en tout cas, que la plupart des souches isolées sont relativement thermorésistantes. Certaines, en effet, résistent à des températures de $+63^{\circ}$.

L'expérience clinique a, malheureusement, confirmé nos recherches bactériologiques. Les laits examinés ont produit, chez les nourrissons qui les ont absorbés, des troubles gastro-intestinaux sérieux, sévères et parfois mortels. Nous avons retrouvé, dans deux selles de malade, que nous avons pu examiner, du staphylocoque doré en abondance. Le lait ingéré dans ce cas mortel était le B. 1.894.

Dans toute analyse bactériologique de lait, il est donc indispensable de compléter la série des épreuves pratiquées ordinairement par une recherche des staphylocoques pathogènes.

\section{(Institut Pasteur, Lille.)}

\section{BIBLIOGRAPHIE}

[1] Milward Bayliss. Studies on the mecanism of vomiting produced by Staphylococcus enterotoxine. Journ. Exp. Med., 72, no 6, décembre ; $1,1,1940$.

[2] B. L. BAmm. Food poisoning produced by Staphylococci. Zhornal mikrobiol. Epidemolo. i. Imm unologii, Moscou, 1942 (1/2), 91-94

[2] B. Slater et J. L. Morris. Food poisoning eaused by hemolytio Staphylococcus in a defense plant. American Journ. Publ. Health., $1944,34,8$, p. $854-856$.

[4] R. L. Worral. Diarrhée et vomissements des enfants. Bristish. Medical, Journ., 1944, 4, 382-865. 
[5] S. Sewit. Bacteriological investigation into the causation of diarrhøa and enteritis in Dublin in 1,942, 1943. Journ. Hyg., 44, I, 1945, p. $37-52$,

[6] G. Mc. L. Lawson et T. S. Englar. An outbreack of food poisoning owing to Staphylococcus aureus. Journ. Bact., 49, I, 1945, p. 19-20.

[7] R. Hermann. Handbuch der Landwertchaftlichen Versuchs, mad Vuter, Suchrings methodick. Rudolph Hermann, 1941. Verlag. J. Neumann, Neudanem und Berlin.

[8] Weinzirl et Velder. American Journ. Publ. Health., t. V, 862, 1915 et t. II, 149, 1921.

[9] Chapman. Journ. Bact., 33, 533, 1937.

[10] Chapman. Journ. Bact., 32, 199, 1936.

[11] R. Kourilsky et P. Mercier. Sur les méthodes de différenciation entre les staphylocoques pathogènes et non pathogènes. Revue d'I mmunologie, t. VII, $\mathrm{n}^{0} 1,2$, p. 53, 1942.

[12] Stone. Proc. Soc. Exp. Biot. Méd., 33, 185, 1935.

[13] Lominski, Iwo et E. Grossfeld. A direct coagulase test for rapid detection of Staphylococcus aureus. Brit. med. Journ., 4, 382, p. $854,1944$.

Formulaire des principaux milieux employés :

A. Bacto-tryptose (Difeo) $\ldots \ldots \ldots \ldots \ldots \ldots \ldots 20 \mathrm{gr}$.

Glucose ......................... 1 gr.

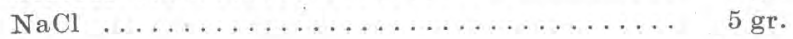

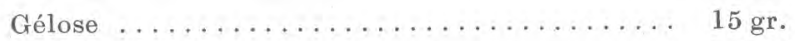

Eau ................... $1.000 \mathrm{~cm}^{3}(p \mathrm{H}=7)$.

B. Extrait de viande de bœuf (Lab. Lemeo) ..... 1 gr.

Protéose-peptone (Difco) .............. $10 \mathrm{gr}$.

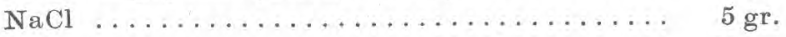

Mannite..................... 10 gr.

Rouge de phénol ................. 0 gr.018

Eau .......................... $1.000 \mathrm{~cm}^{3}(p \mathrm{H}=7,4)$

C. Extrait de viande de bøuf............ 3 gr.

Protéose-peptone (Difeo) ............ 5 gr.

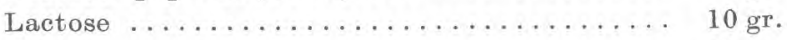

Gélose ................. 15 gr.

Cristal-violet ................... 0 gr. 0033

Eau ........................... $1.000 \mathrm{~cm}^{3}(p \mathrm{H}=6,8)$

D. Gélatine .................. $30 \mathrm{gr}$.

Extrait de viande de bœuf (Lab. Lemco) ...... $30 \mathrm{gr}$.

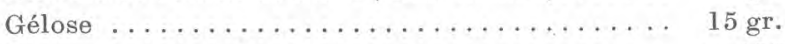

Eau $\ldots \ldots \ldots \ldots \ldots \ldots \ldots \ldots \ldots \ldots \ldots \ldots \ldots \ldots \ldots \ldots \mathrm{cm}^{3}(p \mathrm{H}=6,8)$ 
E. Lactose-Broth :

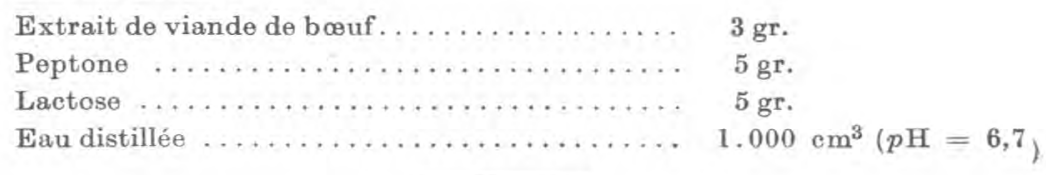

\title{
ÉTUDE COMPARÉE DES FROM AGES DE LIVAROT ET DE PONT-L'ÉVÊQUE
}

\author{
par \\ C. HUREL et G. MOCQUOT
}

Les fromages de Pont-l'Evêque et de Livarot sont fabriqués autour de ces deux petites villes auxquelles ils doivent leur nom, c'est-à-dire respectivement dans le nord et dans le sud du pays d'Auge, en Normandie; les aires de production ne sont du reste pas complètement distinctes : dans la région de Lisieux et au sud de cette ville on trouve dans des fermes voisines les deux types de fromages.

Dans leur pays d'origine, ils sont exclusivement fermiers. Ailleurs, le Pont-l'Evêque seul fait l'objet de quelques fabrications industrielles.

Le Pont-l'Evêque est un fromage carré, ayant le plus souvent $11 \mathrm{~cm}$. de côté; d'un poids voisin de 300 grammes, il demande environ 3 litres de lait par fromage; il est consommable au bout de 4 à 6 semaines; le salage a lieu à la ferme où se poursuit aussi souvent l'affinage : mais une partie de la production est affinée dans une cave coopérative près de Paris.

Le Livarot est cylindrique (diamètre $12 \mathrm{~cm}$., hauteur $5 \mathrm{~cm}$.). Il faut mettre en œuvre 5 à 6 litres de lait pour obtenir un fromage affiné pesant 450 grammes. Le fermier le remet à l'état “ blanc » (âge inférieur à 1 semaine) entre les mains d'un spécialiste : l'affineur, qui se charge du salage et du cavage; l'affinage demande beaucoup de surveillance et de soins et dure environ 3 mois. Après salage, le Livarot passe environ 2 semaines au haloir ; le Pont-l'Evêque, lui, va directement en cave.

Ces deux fromages fermiers à pâte molle sont fabriqués dans la même région naturelle, douée d'une forte individualité provenant d'un ensemble de caractères géologiques, géographiques, climatiques et humains qui lui sont propres.

La seule race laitière exploitée est la race Normande dont le lait est utilisé par les fermiers pour fabriquer indifféremment $d u$ Livarot ou du Pont-l'Evêque.

Dans le présent exposé, nous nous proposons d'établir un paral- 